\title{
Cost of admission for paediatric pneumonia in a setting of human immunodeficiency virus infection
}

\author{
Omolemo P Kitchin, ${ }^{1}$ Cert. Pulm(SA) Paed, Francois Wessels, ${ }^{2}$ Ph.D, Refiloe Masekela, ${ }^{1}$ Cert. Pulm(SA) Paed, Piet Becker, ${ }^{3}$ Ph.D, \\ Robin J Green. 1 Ph.D \\ [1]. Steve Biko Academic Hospital, Department of Paediatrics and Child Health, Pretoria, South Africa \\ [2]. Outcomes Consultants cc. Pretoria, South Africa \\ [3]. Biostatistics Unit - Medical Research Council of South Africa, Pretoria, South Africa
}

\section{Introduction}

Pneumonia in South Africa remains a major public health concern with a number of children admitted each month to hospital for the management of severe pneumonia. The cost of an admission of a patient with community acquired pneumonia (CAP) is seldom considered in the greater scheme of management strategies and it is an important calculation, especially when the cost-efficacy of preventative strategies (such as vaccines) is to be calculated.

The prevalence of CAP is unknown, but there is a global report of 21 million children under 5 years dying annually from acute lower respiratory tract infections (ALRTI). ${ }^{1}$ The prevalence of this condition is estimated to be $2-10$ times greater in Africa and Asia than the United States of America. ${ }^{2}$ Together with diarrhea and malnutrition, ALRTI ranks among the top three causes of death in developing countries. ${ }^{3}$ Pneumonia accounts for nearly one fifth of childhood deaths worldwide, with approximately 2 million children under five dying each year. The majority of deaths occur in Africa and South-East Asia. ${ }^{1,4}$ The Human Immunodeficiency Virus (HIV) and acquired immune deficiency syndrome (AIDS) have had a significant impact on both the prevalence and severity of CAP. In South Africa, there are approximately 80000 new infections annually with 30 to $40 \%$ of admissions to hospital, being HIV related. This results in a disease with a case fatality rate of $15-28 \%{ }^{7}$ The natural consequence of the HIV epidemic, and increase in childhood pneumonia prevalence and severity, is therefore a rise in hospitalization for this condition and consequent increase in disease-related costs. Costs would be dictated by, amongst other, increased numbers of admissions and increased utilization of diagnostic and therapeutic services for more severe disease.

One prevention strategy that has been shown to be highly successful in the USA is routine childhood vaccination with pneumococcal conjugate vaccine. This vaccine has only just been introduced into the immunization schedule in South Africa. Local studies have shown benefit of this vaccine with a reduction of invasive pneumococcal disease by between 65 and 85 percent in HIV-infected and HIV-non- infected children respectively. ${ }^{8}$ However, a cost-efficacy analysis of benefit of such a vaccine, will dictate the real world impact on pneumonia. 
This study has important implications for the following reasons:

The actual cost of a child admitted to hospital with pneumonia has never been determined in a developing country and it is an important pharmaco-economic calculation in a resource poor setting.

This cost is an important quantum when the cost of preventative strategies is to be calculated. This may impact on the calculation of the cost-benefit of routine Pneumococcal 7-valent, 10-valent or 13-valent vaccine for children in South Africa.

The specific increased costs incurred by HIV-infected children should be calculated in order to make a case for early and aggressive implementation of highly active anti-retroviral therapy (HAART), therapeutic and preventative, protocols for children.

\section{Aim}

The aim of this study was to determine the total and individual costs of children admitted to hospital for the management of pneumonia. In addition the study set out to determine the cost differentials for children HIV-infected and those HIV-uninfected and for children admitted to a general paediatric ward or paediatric intensive care unit (PICU). The study set out to measure real costs for a subsidized service (Public sector) and to infer the 'real' value of such services by adjusting for a private fee-for-service setting. This latter calculation was made by means of pricing adjustment.

\section{Methods}

Study design

This was a retrospective observational cross sectional study. The study protocol was approved by the University's ethics committee. A review of each admission of a child for the diagnosis of 'pneumonia', to the Steve Biko Academic Hospital's paediatric pulmonology ward and paediatric intensive care unit (PICU) for the period 1 January 2007 to 31 December 2007, was conducted. Patients with a diagnosis of bronchiolitis were excluded. Each admission had their actual direct costs measured. Direct costs were obtained from the hospital billing file. ${ }^{9}$ These were calculated based on a code of hospital category $\mathrm{H} 2$ - this being a hospitalized patient, partially subsidized by the state, as set out on the Uniform Patient Fee Schedule (UPFS). Public sector fees (included a facility fee, which reflects the overhead costs of providing the environment in which the healthcare service is rendered (tertiary hospital (Level 3)), and a professional fee, which reflects the costs of healthcare professionals rendering the service to the patient), were noted. Costs were calculated per patient per admission and a median cost per diagnosis per ward in each month was calculated. In addition to facility and professional fees, additional actual procedural (including specific testing for HIV-status) and 
therapeutic costs were added. All laboratory blood culture results were noted. The outcome of these admissions was assessed as death in hospital or discharge to home.

All costs also had a theoretical translation added. The actual costs were adjusted to a fee-for-service or private practice rate obtained from the private practice billing manual. ${ }^{10}$

Statistical analysis

A Stata 10 (eStataCorp LP, 4905 Lakeway Drive, College Station, Texas 77845 USA) was used for computations. An analysis of the actual costs was compared for groups using the two-sample Wilcoxon rank-sum (Mann-Whitney) test. A $5 \%$ level of significance was considered statistically significant.

\section{Results}

A total of 200 patients were admitted to the facility for ALRTI during the study period. Sixty eight were excluded because the diagnosis proved to be bronchiolitis (11) or HIV testing was not performed (57). 132 patients, therefore, qualified for data analysis. 86 patients were admitted to the ward and 46 to the PICU. The male to female ratio was $1.45: 1(51: 35)$ in the ward and $1: 1.42(19: 27)$ in the PICU. Thirty three patients (25\%) died, 12 in the ward and 21 in the PICU (Table I).

Table II ( $a$ and $b$ ) reflect the comparison of median costs for HIV-infected patients to HIV-uninfected children in both the ward and PICU and for both Public and Private sectors. The costs of HIV testing were higher for HIV-infected than HIV-uninfected patients in both sectors in the ward $(p=0.02$ for Public and $p=0.01$ for Private respectively). Public and Private adjustment costs for PICU were significant but similar for HIV-infected and uninfected children $(\mathrm{P}=0.002)$. This occurs because an HIV-PCR has to be performed on HIV-ELISA positive children less than 18 months of age to define HIV-infection. $81 \%$ of our cohort required this testing.

The mean cost of an admission to the ward was R3 480.96 for 8.67 days and R6 366.48 for 9.35 days to the PICU. The calculated translational costs for 'private practice' admissions would have been R48 733.92 and R90 724.88, respectively.

PICU radiology costs were statistically similar for both sectors and significantly higher for private sector patients. PICU costs of doctor consultations, nursing fees and antibiotic use in a private sector would have been higher for HIV-infected patients. The public sector costs appear lower, but these are subsized. The antibiotics costs are included in the hospital bed costs in accordance with UPFS. 
HIV-infected patients, admitted to PICU, had on average, more haematological and blood culture tests performed as compared to the HIV-uninfected children and HIV-infected patients in the ward. The mean number of haematological investigations in PICU infected was 16.03 haematological specimen and 1.67 blood culture specimens for HIV-infected while 7.41 haematological specimens and 0.71 blood culture specimens were drawn from HIV-uninfected patients. In patients admitted to the ward, a mean of 6.41 haematological specimens and 0.83 blood culture specimens were collected in HIV-infected patients while a mean of 7.21 haematological specimens and 1.03 blood culture specimens were drawn from HIV-infected patients.

HIV-infected patients had a greater number of positive cultures for bacteria as compared to their HIV-uninfected counterparts. This was noted in both the ward and PICU.

The total mortality was $25 \%$ (13.9\% and $36.9 \%$ in the ward and PICU respectively). Survival estimates revealed a significant difference between HIV-infected patients and HIV-uninfected in the ward $(p=0.03)$ (Figure 1), while for patients admitted to the PICU, there was no difference between the two groups $(p=0.80)$ (figure 2$)$.

\section{Discussion}

Children admitted to hospital for the management of severe pneumonia cost the state a significant amount of money each year. Both HIV-infected and HIV-uninfected children are still admitted to hospital and PICU with pneumonia. The total number of HIV-uninfected children with severe and very severe pneumonia (28 to the ward and 15 to PICU respectively) during the study year (2007), suggest that even in the absence of HIV-infection, pneumonia is still a common condition of children. On average there are just more than 2 ward admissions and 1 PICU admission per month. In addition to the usual risk factors for very severe pneumonia described in Western studies, ${ }^{11}$ it is important to include HIV-infection, especially for children, from the developing world. The mortality from pneumonia occurs in HIV-infected severe (ward) and both HIV-uninfected and infected very severe pneumonia (PICU). This has been well described. ${ }^{12,13}$ However what has emerged from this study is that once very severe pneumonia occurs the protective effect of not having HIV-infection is lost for early mortality but HIV-infected children continue to die well into PICU stay. The reason for this late mortality phenomenon is poorly understood but immune compromise has been listed as a reason for hospital-acquired pneumonia. $^{14}$ Many reasons for similar early PICU mortality have been suggested but it is likely that the host inflammatory response takes over as a major driver of disease in very severe pneumonia. ${ }^{15}$

In addition HIV-infected children admitted to hospital with pneumonia cost more, probably not surprisingly, but seldom considered. The real, unsubsidized costs borne by the public sector are probably reflected by the private sector costs. This calculation is 
important when the true cost-benefit ratio of preventative strategies is to be calculated. Blood culture and microbiology costs were not statistically significantly different between HIV-infected and HIV-uninfected children in both sectors since all children have an initial microbiological screen and in our setting regular and routine microbiological cultures are discouraged. Patients admitted to the PICU had significantly more haematological investigations performed, irrespective of HIV status, confirming a sicker population group. HIV-infected patients admitted to the PICU incurred higher hospital bed costs (as reflected by a longer duration of stay) as compared to HIV-uninfected patients in both sectors. The duration of hospitalization was 1.8 days longer in the ward and 5.7 days longer in PICU respectively. The pattern of organisms identified from blood culture on these children is similar to that described from other similar African settings. ${ }^{16}$ Lack of positive yield on blood culture is a universally acknowledged phenomenon in childhood pneumonia. $^{17}$ The greater likelihood of culturing an organism from blood specimens in HIV-infected children has also been previously described. ${ }^{17}$ This study was conducted before a routine policy of sampling for Pneumocystis jerovicii and Cytomegalo Virus was introduced.

\section{Conclusions}

Children admitted for pneumonia cost the public sector a significant amount of money each year. HIV-infected children cost more. These costs need to be borne in mind when preventative campaigns are embarked on.

The Public sector spends a significant amount of money in procuring antiretroviral treatment for HIV-infected patients and treating consequences of uncontained HIV disease. These costs are set to escalate unless authorities and medical personnel alike reenforce preventative strategies such as reducing mother to child transmission (MTCT) of the $\mathrm{HI}$ virus and vaccines that prevent infectious diseases. This should be coupled with reducing new infections in the parents of these children. Only when all preventative strategies are utilized in conjunction, will the burden of pneumonia and its attendant costs come down. This study adds to the mounting evidence that childhood pneumonia is a costly illness ${ }^{18}$ and that all efforts should be focused on preventing its occurrence.

\section{References:}

1.Jeena PM, McNally LM, King S, Davis M, Lewis $H$. The epidemiology and aetiology of paediatric community acquired pneumonia. S Afr Resp J 2006;12:56-61

2. Williams BG, Gouws E, Boschito-Pinto C, Bryce J, Dye C. Estimates of world-wide distribution of child deaths from acute respiratory infections. Lancet Infect Dis 2002;2:25-32

3. Nascimento-Carvalho CMC. Etiology of childhood community acquired pneumonia and its implications for vaccination.

Brit J Infect Dis 2001;5:87-97 
4. Mulholland K. Magnitude of the problem of childhood pneumonia. Lancet 1999;354:590-2

5. Jeena $\mathrm{P}$. The role of HIV infection in acute respiratory infections among children in sub-Saharan Africa. Int Tuberc Lung

Dis 2005;9:708-15

6. Graham SM, Gibb DM. HIV disease and respiratory infection in children. Br Med Bull 2002;6:133-150

7. Shann F, Gratten M, Germer S, Linnemann V, Hazlett D, Payne R. The etiology of pneumonia in children in Goroka Hospital, Papua New Guinea. Lancet 1984;2:537-41

8. Klugman KP, Madhi SA, Huebner RE, Kohberger R, Mbelle N, Pierce N. A trial of 9 valent Pneumococcal conjugate vaccine in children with and those without HIV infection. N Eng J Med 2003;349:1341-48

9. Department of Health. Republic of South Africa. Uniform patient fee schedule for paying patients attending public facilities, 2007

10. Council for Medical Schemes. National Health Reference Price List, 2007. Pretoria: Council for Medical Schemes, 2007 www. medicalschemes.com/nrpl/nrplpublications. aspx?Catld=26 Accessed 26/08/2010

11. Tiewsoh K, Lodha R, Pandey RM, Broor S, Kalaivani M, Kabra SK. Factors determining the outcome of children hospitalized with severe pneumonia. BMC Pediatr. 2009 Feb 23;9:15

12. Ferrand RA, Bandason T, Musvaire $\mathrm{P}$, et al. Causes of acute hospitalization in adolescence: burden and spectrum of HIV-related morbidity in a country with an early-onset and severe HIV epidemic: a prospective survey. $\underline{\text { PLoS Med. }}$ 2010;7(2):e1000178

13. Sigaúque B, Roca A, Bassat Q , et al. Severe pneumonia in Mozambican young children: clinical and radiological characteristics and risk factors. J Trop Pediatr. 2009;55(6):379-87

14. Morrow B, Argent AC, Jeena PM, Green RJ. Guideline for the diagnosis, prevention and treatment of paediatric ventilator-associated pneumonia. S Afr Med J 2009; 99: 255-267

16. Nantanda R, Hildenwall H, Peterson S, Kaddu-Mulindwa D, Kalyesubula I, Tumwine JK. Bacterial aetiology and outcome in children with severe pneumonia in Uganda. Ann Trop Paediatr. 2008;28(4):253-60

17. Zar HJ, Jeena P, Argent A et al. Diagnosis and management of community-acquired pneumonia in childhood-South African Thoracic Society Guidelines. SAMJ 2005;95(12):975-990

18. Madsen $\mathrm{HO}, \underline{\mathrm{Haneh} ø j} \mathrm{M}, \underline{\text { Das AR }}$ et al. Costing of severe pneumonia in hospitalized infants and children aged 2-36 months, at a secondary and tertiary level hospital of a not- for-profit organization. Trop Med Int Health. 2009;14(10):1315-22 
Table I. Overview of the patient profile and outcome of children admitted with pneumonia. n=132, IQR= Inter Quartile Range

\begin{tabular}{|l|l|l|}
\hline & Ward & PICU \\
\hline HIV-infected & $58 / 86(67 \%)$ & $31 / 46(67 \%)$ \\
\hline Deaths - Otal & $12 / 86(14 \%)$ & $21 / 46(46 \%)$ \\
\hline Deaths -HIV infected & $12 / 86(14 \%)$ & $17 / 46(36.9 \%)$ \\
\hline Deaths - HIV uninfected & $0 / 86(0 \%)$ & $4 / 46(8.6 \%)$ \\
\hline Length of stay in days Median(IQR) & $7(5-12)$ & $6.5(3-14)$ \\
\hline Length Of stay HIV-infected in days & $8(4-12)$ & $9(5-18)$ \\
Median(IQR) & & $3(2-7)$ \\
Length of stay HIV-uninfected in days & $6.5(5-8)$ & \\
Median(IQR) & & \\
\hline
\end{tabular}

Table II(a) Median costs per patient (in Rands) in the Ward (Public and Private) in each category.

\begin{tabular}{|c|c|c|c|c|c|c|}
\hline \multirow[t]{2}{*}{ Cost centre } & \multicolumn{2}{|c|}{ Public } & \multirow[t]{2}{*}{$P$ value * } & \multicolumn{2}{|c|}{ Private } & \multirow[t]{2}{*}{$P$ value * } \\
\hline & HIV - infected & $\begin{array}{l}\text { HIV } \\
\text { uninfected }\end{array}$ & & HIV - infected & $\begin{array}{l}\text { HIV } \\
\text { uninfected }\end{array}$ & \\
\hline HIV testing & 365 & 87.2 & 0.02 & 578.2 & 108.7 & 0.01 \\
\hline Blood culture & 90.4 & 90.4 & 0.54 & 280.7 & 280.7 & 0.59 \\
\hline $\begin{array}{l}\text { Sputum } \\
\text { microbiology }\end{array}$ & 230 & 230 & 0.18 & 315.30 & 315.30 & 0.18 \\
\hline $\begin{array}{l}\text { Haematological } \\
\text { testing }\end{array}$ & 478.3 & 264.95 & 0.01 & 766.2 & 522.15 & 0.02 \\
\hline Hospital bed & 1388.28 & 1115.89 & 0.23 & 9368.8 & 7612.15 & 0.23 \\
\hline Radiology & 247 & 247 & 0.25 & 413 & 413 & 0.25 \\
\hline Antibiotics & 0 & 0 & 0.20 & 2108 & 1278 & 0.23 \\
\hline
\end{tabular}

*Statistical significance for comparison of HIV-infected patients to HIV-uninfected patients 
Table II(b) Median costs per patient (in Rands) in the PICU (Public and Private) in each category.

\begin{tabular}{|c|c|c|c|c|c|c|}
\hline \multirow[t]{2}{*}{ Cost centre } & \multicolumn{2}{|c|}{ Public } & \multirow[t]{2}{*}{ P value* } & \multicolumn{2}{|c|}{ Private } & \multirow[t]{2}{*}{ P value } \\
\hline & HIV - infected & $\begin{array}{l}\text { HIV } \\
\text { uninfected }\end{array}$ & & HIV - infected & $\begin{array}{l}\text { HIV } \\
\text { uninfected }\end{array}$ & \\
\hline HIV test & 365 & 87.2 & 0.002 & 578.2 & 108.7 & 0.002 \\
\hline Blood cultures & 90.4 & 90.4 & 0.42 & 280.7 & 280.7 & 0.42 \\
\hline $\begin{array}{l}\text { Microbiology- } \\
\text { sputum }\end{array}$ & 230 & 230 & 0.54 & 315.30 & 315.30 & 0.54 \\
\hline $\begin{array}{l}\text { Haematological } \\
\text { testing }\end{array}$ & 828.2 & 570.7 & 0.17 & 1418.2 & 990.1 & 0.18 \\
\hline Hospital bed & 3060 & 1020 & 0.01 & 35156.7 & 11718.9 & 0.01 \\
\hline Radiology & 365.8 & 365.8 & 0.04 & 498.8 & 498.8 & 0.04 \\
\hline Antibiotics & 0 & 0 & 0.11 & 2844 & 588 & 0.01 \\
\hline
\end{tabular}

"Statistical significance for comparis on of HIV-infected patients to HIV-uninfected patients 